\title{
Research Article \\ Research on Image-Based Movement Accuracy Monitoring of Aerobics
}

\author{
Ting Feng $(1)$ \\ Ministry of Sports and Public Art, Zhengzhou University of Aeronautics, Zhengzhou, Henan 450015, China \\ Correspondence should be addressed to Ting Feng; fengting@zua.edu.cn
}

Received 15 June 2020; Accepted 30 June 2020; Published 28 October 2020

Guest Editor: S. A. Edalatpanah

Copyright (C) 2020 Ting Feng. This is an open access article distributed under the Creative Commons Attribution License, which permits unrestricted use, distribution, and reproduction in any medium, provided the original work is properly cited.

\begin{abstract}
The movement accuracy monitoring of aerobics is mostly performed through three-dimensional reconstruction of aerobic movements. The feature extraction of aerobics is based on the optimal classification decision function, which extracts all the features of aerobics and thus reduces the accuracy of aerobics monitoring. In order to extract the aerobic motion in the background with higher accuracy, a new image-based monitoring method is proposed. First, the Kinect depth image acquisition method is used to preprocess the image, and then Hog $3 \mathrm{D}$ is used to extract aerobic movement features and analyze the extraction results. This new method solves the problem of video content classification in aerobics precision monitoring. The Adaboost method in probability statistics is used to identify the accuracy of aerobic motions. This paper uses probability function to link the postures of aerobics and forms an action sequence and its ergodic function to take the maximum value of an aerobic exercise. The accuracy of aerobics is monitored by using the method of level by level proportional example. The experimental results show that this method can effectively improve the accuracy of aerobic track monitoring, reduce the energy consumption of aerobic movement accuracy monitoring, and has good use value.
\end{abstract}

\section{Introduction}

With the development of the modern society, the demand for fitness is continuously growing among people. Sports consumption is seen as a kind of new fashion. As a form of aerobic exercise, calisthenic moves can effectively improve body quality, cardiopulmonary function, and muscular endurance and help the human body to achieve the optimal state. Different from other aerobic exercises, calisthenic exercise is exquisite and at the same time gives people the enjoyment of art, therefore getting more and more attention from people. Detecting the accuracy of calisthenic movements will allow us to improve their effectiveness. However, at present, most of the aerobic movement accuracy detection methods are based on the selection of aerobic movement characteristics by using the wavelet threshold denoising method. First, the features are classified, and then the optimal aerobic movement characteristic parameters are selected by using the supporting direction machine method so as to detect the accuracy of aerobic movements. In this method, it is difficult to overcome the fixed attenuation or oscillation problem and also distinguish the aerobic movements with high similarity. Also, this method has strong repercussions in the industry. With the increasing attention to calisthenics, the research on calisthenic movement accuracy detection has gradually deepened, producing fruitful results.

Liang [1] proposed a method for aerobic movement monitoring based on body signals. In this method, sensor nodes are installed on the human body, body aerobics signals are collected, and then based on the coupled hidden Markov model, bodybuilding movements are constructed. The random process model is used for data linking, and multiple statistical analyses are carried out on the aerobic movement based on the corresponding data link and the sensor node information. This method describes the characteristics of aerobic exercise and maps the coordination of various parts of the body in aerobics through the transmission between different data links. Based on the probabilistic neural network and the adaptive fuzzy clustering algorithm, the 
motion characteristics of calisthenics are extracted, and the motion accuracy of calisthenics is monitored by inertial signals. Because in this method the sensor node needs to be installed on the body, it affects the comfort of calisthenics, and it is difficult to guarantee the effect of calisthenics. The monitoring method of aerobics movement accuracy proposed in reference [2] combines the action recognition method of RP ccall and RP cceach compression classification with compressed sensing and sparse representation theory, combines sensing signal compression with aerobics action recognition, establishes a recognition model of aerobics movement pattern based on sparse representation of compressed data, and realizes it by using the distribution of sparse coefficient. Aerobics action recognition is done so as to realize the precision monitoring of aerobics. Because this method pays too much attention to the identification and ignores the monitoring of calisthenic movements, the accuracy of calisthenic movement precision monitoring is not high. In [3], Song and $\mathrm{Wu}$ proposed an aerobic movement accuracy monitoring method. Based on distributed compressed perception and joint sparse representation movement identification method, the complex movement identification problem with multiple sensors is transformed into a linear regression problem with a bias toward mathematics to solve the problem by building a sparse description model suitable for movement identification. Thus, the progress detection of calisthenic movements can be realized. However, the monitoring process of this method is complicated and thus time-consuming. For other literature, please refer to [4-9].

In this paper, we propose a new method for aerobic movement accuracy monitoring based on calisthenic motion images.

\section{Monitoring of Aerobics Movement Precision Based on Image}

2.1. Image-Based Aerobic Movement Feature Extraction. Separating a specific action from the whole background is key to extract the action characteristics of aerobics and then to complete the detection of the action characteristics. In this paper, the Kinect depth image preprocessing method is adopted to extract aerobic movements from the background through the depth dimension data of the depth image.

In the depth image sequence supplied by the depth data stream, the set pixel represents the linear distance between the coordinate $(x, y)$ of the specific object and the closest part of the lens plane of the depth data acquisition device to the lens plane within the field of view of the depth data acquisition device. The depth image data stream obtained using Kinect can reduce the amount of computation in the preprocessing stage.

In order to improve the accuracy of calisthenic action accuracy monitoring, the feature vector should have good identification. In this paper, local regional features are extracted by the Hog3D method, and the specific feature extraction process is shown in Figure 1.

When the 3D gradient direction is expressed, the expression direction of the characteristic points of the aerobic

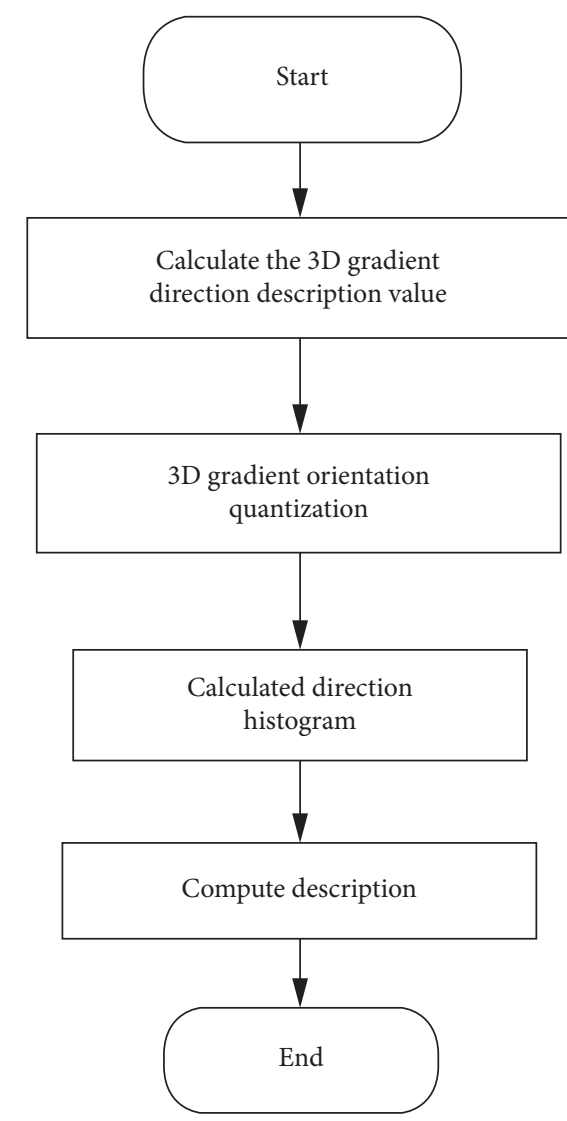

FIGURE 1: Extraction process of aerobic movement characteristics by using the Hog3D method.

movement and its adjacent areas can be calculated from different dimensions. There are $N$ scales, in which $\sigma_{x} y$ represents the scale factor of space and $\sigma_{t}$ represents the scale factor of time, and the number of scale factors is expressed as follows:

$$
z=\sum_{i=0}^{N-1} \sum_{j=0}^{N-1} \sigma_{x y}^{-2 i} \sigma_{t}^{-j}
$$

In order to speed up the operation of the average gradient vector, the integral image is introduced.

A video sequence is set as follows: $v(x, y, t), v_{\delta x}, v_{\delta y}$, and $v_{\delta t}$, respectively. The partial derivative of the video sequence at $x, y$, and $t$ and then $v_{\delta x}$ integral video is expressed as follows:

$$
i v_{\delta x}(x, y, t)=\sum_{x^{\prime} \leq x, y^{\prime} \leq y, t^{\prime} \leq t} v_{\delta x}\left(x^{\prime}, y^{\prime}, t^{\prime}\right) .
$$

Similarly, the integral video expressions of $v_{\delta y}$ and $v_{\delta t}$ are obtained as follows:

$$
\begin{aligned}
i v_{\delta y}(x, y, t) & =\sum_{x^{\prime} \leq x, y^{\prime} \leq y, t^{\prime} \leq t} v_{\delta y}\left(x^{\prime}, y^{\prime}, t^{\prime}\right), \\
i v_{\delta t}(x, y, t) & =\sum_{x^{\prime} \leq x, y^{\prime} \leq y, t^{\prime} \leq t} v_{\delta t}\left(x^{\prime}, y^{\prime}, t^{\prime}\right) .
\end{aligned}
$$

For any three-dimensional space, cuboid $b=(x, y$, $t, w, h, l)^{t}$, where $(x, y, t)^{t}$ represents the position of aerobic 
action feature points; $w$ stands for three-dimensional emptiness, $h$ is the height of the three-dimensional space; and $l$ stands for the length of the three-dimensional space. The average gradient is set as follows:

$$
\bar{g}_{b}=\left(\bar{g}_{b \delta x}, \bar{g}_{b \delta y}, \bar{g}_{b \delta t}\right)^{T},
$$

where $\bar{g}_{b \delta x}, \bar{g}_{b \delta y}$, and $\bar{g}_{b \delta t}$ represent the width, height, and length of the three-dimensional space, respectively, and the average gradient is represented in degrees. $\bar{g}_{b \delta x}$ is calculated from the following formula:

$$
\begin{aligned}
\bar{g}_{b \delta x}= & {\left[i v_{\delta x}(x+w, y+h, t+l)-i v_{\delta x}(x, y+h, t+l)\right.} \\
& \left.-i v_{\delta x}(x+w, y, t+l)+i v_{\delta x}(x, y, t+l)\right] \\
& -\left[i v_{\delta x}(x+w, y+h, t)-i v_{\delta x}(x, y+h, t)\right. \\
& -i v_{\delta x}(x+w, y, t)+i v_{\delta x}(x, y, t] .
\end{aligned}
$$

Similarly, formula (5) can be used to calculate $\bar{g}_{b \delta y}$ and $\bar{g}_{b \delta t}$.

The 2D aerobic image gradient direction is quantified, and the histogram of the gradient direction is regarded as a circle. The circle is divided into $N$ regions, and each straight area is approximately described by multiple feature points.

Set $\bar{g}_{b}$ to represent the average eigenvector of the multidimensional space; the process of mapping $\bar{g}_{b}$ to the spatial region is represented by matrix multiplication. Set $P=\left(p_{1}, p_{2}, \ldots, p_{n}\right)^{T}$ to represent the center position of $n$ faces, where $p_{i}=\left(x_{i}, y_{i}, t_{i}\right)^{T}$. If $q b=\widehat{q}_{b-t}$ is a mapping of $g b$, then there is a relation:

$$
\widehat{q}_{b}=\left(\widehat{q}_{b 1}, \widehat{q}_{b 2}, \ldots, \widehat{q}_{b n}\right)^{T}=\frac{P \cdot \bar{g}_{b}}{\left\|\bar{g}_{b}\right\|_{2}} .
$$

Type: $\widehat{q}_{b i}=\left\|p_{i}\right\|_{2} \cdot \cos \angle\left(p_{i} \bar{g}_{b}\right)=\left\|\bar{g}_{b}\right\|_{2}^{-1} \cdot p_{i}^{T} \cdot \bar{g}_{b}$, needs to be done in the $P$ projection vector threshold in order to ensure the accuracy of the $\widehat{q}_{b}$ range, compare each center below $p_{i}$ and $p_{j}$, and get the threshold $t=p_{i}^{T} \cdot p_{j}$. The vector after threshold quantization can be expressed as $q b=\widehat{q}_{b-t}$. When $q b<0$, the vector is assigned to 0 , and the projection gradient is obtained:

$$
q_{b}=\frac{\left\|\bar{g}_{b}\right\|_{2} \cdot \widehat{q}_{b}}{\left\|\hat{q}_{b}\right\|_{2}} .
$$

Set the sampling point $s=\left(x_{s}, y_{s}, t_{s}, \sigma_{s}, \tau_{s}\right)^{T}$, where $\left(x_{s}, y_{s}, t_{s}\right)^{T}$ represents the set of projected feature points, $\sigma_{s}$ represents the projected time gradient, and $\tau_{s}$ is the projection space gradient. The description value $d_{s}$ at the sampling point $s$ is calculated by the characteristic region $r_{s}=\left(x_{r}, y_{r}, t_{r}, w_{r}, h_{r}, l_{r}\right)^{T}$. Formula (8) is used to calculate the direction histogram to form a single aerobic action feature vector $d_{s}=\left(d_{1}, d_{2}, \ldots, d_{M^{2} N}\right)^{T}$ :

$$
h_{c}=\sum_{i=1}^{s^{3}} q_{b i} \text {. }
$$

Through the above discussion, the aerobics movements were extracted from the background, the images were preprocessed by using the Kinect depth image acquisition method, and then the aerobics movement features were extracted by HOG3D. The movement characteristics of calisthenics are extracted by using Hog3D.

2.2. Image-Based Aerobic Movement Accuracy Analysis and Monitoring. Calisthenic motion accuracy analysis and monitoring can be converted into a video content classification problem. The sequence to be examined is matched with a precalibrated reference sequence representing a typical motion to make it robust to slight characteristic changes in space and time scales.

In this paper, the Adaboost method in probability statistics is used to identify the aerobic movement accuracy. By defining each pose as a state of the aerobic movement to be tested and at the same time combining each state of the aerobic movement with the probability formula, the joint probability of each calisthenic action sequence is calculated, and the maximum probability is the final classification standard of the calisthenic action.

By one-to-one correspondence between the weak classifier and the aerobic action features extracted in Section 2.1, the prototype of the weak classifier is expressed as follows:

$$
h_{j}(x)= \begin{cases}\alpha_{1}, & f_{j}(x) \leq \theta_{j}, \\ \alpha_{2}, & \text { else }\end{cases}
$$

where $x$ represents the window to be checked, $h_{j}(x)$ is a function of volume eigenvalue, $\theta-j$ represents a trained eigenvalue threshold, and $\alpha_{1}$ and $\alpha_{2}$ represent the decimals in the $[-1,1]$ range, indicating the result of the classification. In general, if the classification is correct, then $\left|\alpha_{1}\right|=\left|\alpha_{2}\right|=1$.

After the identification and analysis of calisthenic motion images, the accuracy of calisthenic motion is monitored. In this paper, the author chooses the method of layer by layer equal ratio to monitor the precision of aerobics The specific process is described below.

Suppose that $\mathrm{V}$ represents the aerobics action video to be inspected, and the size is $\mathrm{W} * \mathrm{H} * \mathrm{~L}$, where $\mathrm{W}$ and $\mathrm{H}$ represent the width and height of the frame image and $\mathrm{L}$ represents the number of frames. Set the size of the current monitoring window as Winsize $\times$ Winsize $\times 40$, and the initial value of Winsize is 64 and the scale of serial port amplification is 1.2. The number of window magnification is calculated by the following formula:

$$
T=\left\lfloor\log 1.2 \frac{\min W, H}{64}\right\rfloor .
$$

When $i=1 / T$, the whole aerobic action video sequence $V$ is traversed through the detection window with the size of Winsize $\times$ Winsize $\times 40$ to calculate the number of Windows to be detected:

$$
\text { num }=(W-\text { Winsize }) \times(H-\text { Winsize }) \times(L-40) .
$$

The current window is monitored by a classifier. If the monitoring results are correct, the relevant parameters will be output and marked in the video. Otherwise, continue monitoring the next window. Then, some main parameters 
of the volume characteristics corresponding to the weak classifier are updated.

The weight of two cuboids in the volume feature is expressed as follows:

$$
\begin{aligned}
& w_{1}^{\prime}=\frac{w_{1}}{\text { Winsize } \times \text { Winsize }}, \\
& w_{2}^{\prime}=\frac{w_{2}}{\text { Winsize } \times \text { Winsize }},
\end{aligned}
$$

where $w_{1}$ and $\mathrm{w}_{2}$ represent the initial weight. The coordinates of the upper left corner of the first frame of the two cuboids with volume characteristics are as follows:

$$
\begin{aligned}
& \left(x_{1}^{\prime}, y_{1}^{\prime}, t_{1}^{\prime}\right)=\left(\text { scale } \times x_{1}, \text { scale } \times y_{1}, t_{1}\right), \\
& \left(x_{2}^{\prime}, y_{2}^{\prime}, t_{2}^{\prime}\right)=\left(\text { scale } \times x_{2}, \text { scale } \times y_{2}, t_{2}\right) .
\end{aligned}
$$

By multiplying the width and length of the two cuboids with volume characteristics by scale, respectively, the imagebased aerobic movement accuracy analysis and monitoring are completed.

\section{Experimental Results and Analysis}

In order to prove the effectiveness of the image-based aerobics movement accuracy monitoring method proposed in this paper, taking Intel p42g processor as the hardware environment and Matlab2008a as the platform, the comparison method is used to compare the aerobics movement accuracy monitoring method proposed in this paper with those in references [8] and [9], and the experiment is completed.

Firstly, the target azimuth coordinates of the aerobic movement were monitored according to three kinds of aerobic movement accuracy monitoring methods, and the comparative monitoring results are shown in Figures 2 and 3 .

It can be found from Figures 2 and 3, the maximum error of the proposed method does not exceed 1, indicating that the prediction accuracy of the proposed method meets the monitoring requirements of the aerobic movement accuracy. If there are not many missing frames, the track of aerobic movements can be monitored.

Then, the aerobic movement accuracy monitoring experiment was conducted and the accuracy of aerobic movement track monitoring was compared with the proposed method and reference [8-9]. The comparison results are shown in Figure 4.

It can be seen from Figure 4 that the proposed method has higher monitoring accuracy of calisthenic action trajectory than the methods proposed in reference [8-9], indicating that the proposed method can better improve the accuracy of monitoring of calisthenic action accuracy.

Finally, the energy consumption of the three aerobic movement accuracy monitoring methods is compared. The calculation method of energy consumption (unit: J) is as follows:

$$
Q=I V H
$$

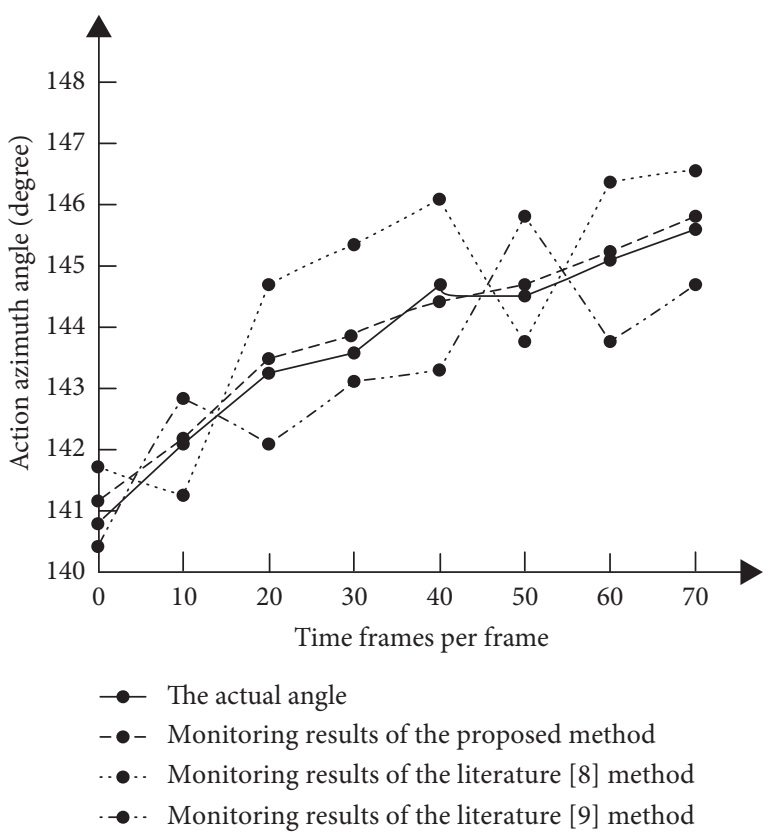

FIGURE 2: Monitoring angle and actual angle of aerobic movement azimuth angle.

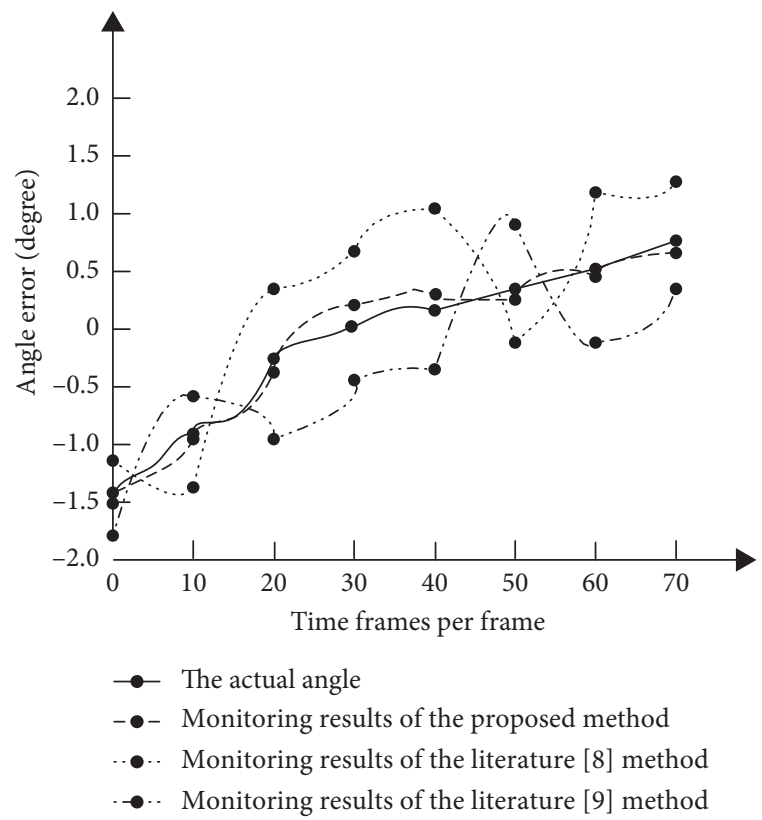

FIGURE 3: Monitoring angle error curves of aerobic movement azimuth angle.

where $I$ represents the operating current during precision monitoring, $V$ represents the supply voltage; and $H$ stands for the precision monitoring time. The energy consumption comparison results of the three methods are shown in Figure 5 .

It can be seen from Figure 5 that the proposed method can effectively reduce the energy consumption of aerobic movement progress monitoring. In Figure 5, the broken line representing the energy consumption of the proposed 


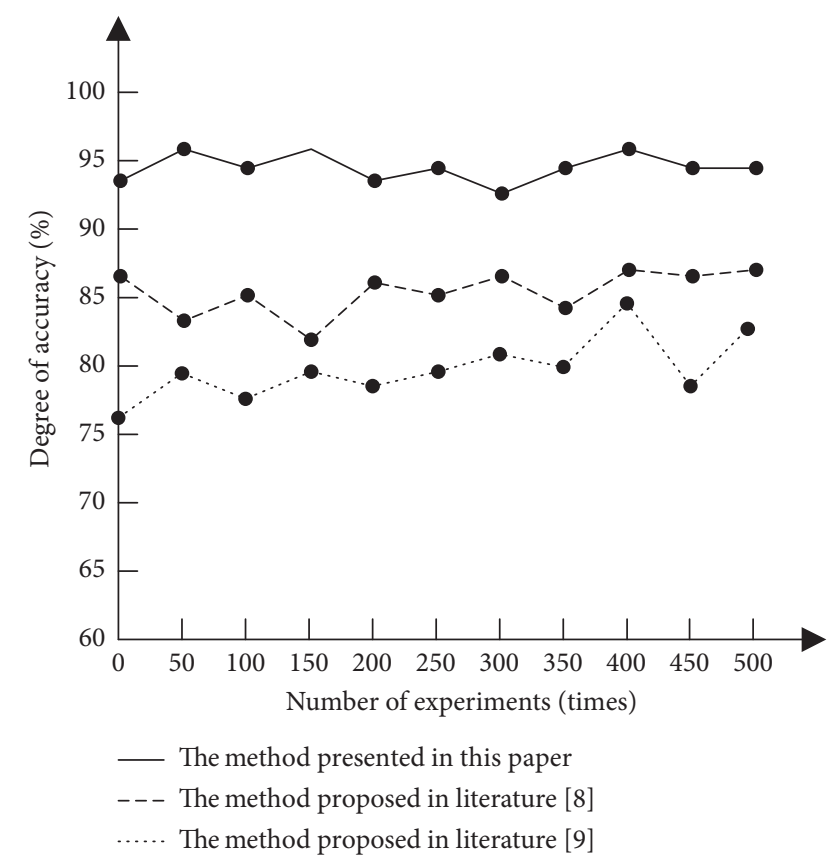

FIGURE 4: Accuracy comparison of three methods for aerobic movement track monitoring.

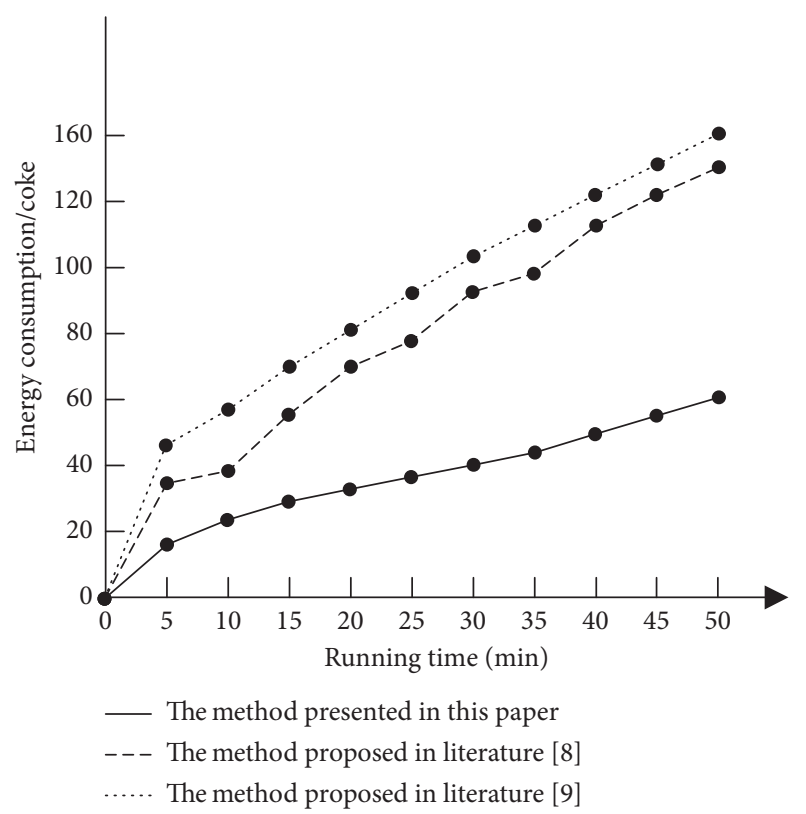

FIGURE 5: Energy consumption comparison of three real-time monitoring feedback methods.

method appears to be an approximately straight line, indicating that the operating energy consumption of the proposed method is more stable, thus indicating that the proposed method is more stable in the accuracy monitoring of aerobic movements. To sum up, the method proposed in this paper can effectively improve the accuracy of calisthenic movement accuracy monitoring, reduce the accuracy monitoring error, decrease energy consumption, and has good use value.

\section{Conclusion}

The accuracy of calisthenic movement monitoring has a great influence on the results of calisthenic training. This paper proposes an image-based accuracy monitoring method for calisthenic movements. The experimental results show that the proposed method can effectively improve the accuracy of calisthenic movement monitoring and play a potential role in the development of calisthenics.

\section{Data Availability}

The data used to support the findings of this study are included within the article.

\section{Disclosure}

The abstract of the manuscript was presented in "Research on Image Precision Monitoring of Aerobics Movement."

\section{Conflicts of Interest}

The author declares that there are no conflicts of interest.

\section{Acknowledgments}

This research was supported by Henan Humanities and Social Sciences Research Project (2018-zzjh-596), the National Natural Science Foundation of China (11226337, 11501525, 11961082, 11801528, 61571104, and 41906003), Aeronautical Science Foundation of China (2017ZD55014), Basic Research Projects of Key Scientific Research Projects Plan in Henan Higher Education Institutions (20zx003), Project of Youth Backbone Teachers of Colleges and Universities of Henan Province (2019GGJS100 and 2019GGJS176), Key Projects of Colleges and Universities in Henan (20A520009 and 21A510013), Sichuan Science and Technology Program (2019YJ0357), and Young Backbone Teachers Training Program of Henan Province (2020GGJS240).

\section{References}

[1] J. Liang, "Heterogeneous data mining model of national fitness benefit index diversification," Bulletin of Science and Technology, vol. 31, no. 8, pp. 108-110, 2015.

[2] X. Wang, J. Zhang, L. Wu et al., "A key pose frame extraction approach combined with trajectory from weightlifting video," Journal of Graphics, vol. 35, no. 2, pp. 256-261, 2014.

[3] L. Min, Y. Song, B. Wu et al., "Three-dimension human motion tracking based improved particle filter on Beowulf cluster system," Computer Engineering and Applications, vol. 51, no. 14, pp. 17-22, 2015.

[4] H. Gao, "Basketball player jump shot video motion precision three-dimensional monitoring simulation research," Computer Simulation, vol. 33, no. 10, pp. 183-186, 2016.

[5] R. Fu, W. Cheng, M. Zhang et al., "Driver mouth behavior recognition and hierarchical prewarning based on dynamic matching model," Automotive Engineering, vol. 37, no. 9, pp. 1095-1102, 2015. 
[6] G. Chang, H. Wang, L. Rong et al., "Video moving target trail tracking algorithm based on state dependence detection," Modern Electronics Technique, vol. 39, no. 7, pp. 51-56, 2016.

[7] Y. Jiang and Q. Zhang, "Prediction algorithm of dynamic trajectory based on weighted grey model," Journal of Computer Applications, vol. 36, no. 5, pp. 1336-1340, 2016.

[8] K. Zhang, S. Yang, and G. Wang, "Measurement error suppressing method in infrared seeker," Computer Measurement Control, vol. 23, no. 6, pp. 2030-2032, 2015.

[9] Z. Wang and S. Yang, "Using Kalman to research tracking and prediction of moving object," Science Technology and Engineering, vol. 14, no. 29, pp. 234-237, 2014. 